\title{
Intermittent Complete Heart Block in Acute Calculous Cholecystitis
}

\author{
MAŠA SORIĆ, WILMA MILETIĆ, SEAD ŽIGA, VLATKO GRABOVAC \\ Department of Emergency Medicine, University Hospital Dubrava, Zagreb, Croatia \\ Corresponding author: \\ Maša Sorić \\ Department of Emergency Medicine, University Hospital Dubrava, G. Šušak Avenue 6, 10000 Zagreb, Croatia \\ Phone: +385917849326 \\ E-mail:masa.soric@kbd.hr
}

\section{ABSTRACT}

The aim of this case report is to present a case of a 24-year old male patient with known cholelithiasis who was presented to the emergency department with acute calculous cholecystitis. The patient was initially in sinus rhythm, but during observation converted into a complete heart block. A temporary heart pacemaker was inserted prior to an emergency laparoscopic cholecystectomy. Postoperatively, the patient converted back to sinus rhythm and made a complete recovery. Considering the patient's age, normal cardiac workup and that his arrhythmia disappeared after the removal of his gallbladder, it was postulated that this patient had a case of cardio-biliary reflex. Special attention must be given to patients with acute cholecystitis and electrocardiographic changes. Acute cholecystitis is a treatable cause of a newly diagnosed atrioventricular block, thus immediate cholecystectomy should not be deferred.

Key words: Atrioventricular block, cholecystitis, gallstones, cardio-biliary reflex

\section{INTRODUCTION}

Acute cholecystitis (inflammation of the gallbladder) is most commonly caused by gallstones. Acute cholecystitis develops in $1-3 \%$ of patients with symptomatic gallstones and is diagnosed on the basis of symptoms and signs of inflammation in patients with localized peritonitis in the right upper quadrant. (1) Abdominal ultrasound is the investigation of choice in suspected acute cholecystitis. Sonograms typically show pericholecystic fluid (fluid around the gallbladder), gallbladder distension, gallbladder wall oedema and gallstones. Immediate measures should be taken to rest the gallbladder which will subdue the inflammatory process in most patients. (2) Patients should be fasted, rehydrated with intravenous fluids, given oxygen therapy and adequate analgesia. Approximately $20 \%$ of patients with acute cholecystitis need emergency surgery, which is indicated if the patient's condition deteriorates or when generalised peritonitis or emphysematous cholecystitis is present. These features suggest gangrene or perforation of the gallbladder. (2) A third-degree atrioventricular (AV) block or complete heart block is characterized by independent atrial and ventricular activity. The hemodynamic consequences depend on the ventricular rate and the presence of underlying heart disease. A complete heart block is most commonly caused by coronary artery disease or by degeneration of the cardiac conduction system. (3) In this case report we would like to present the case of a 24-year old male patient with acute calculous cholecystitis, which was complicated by an intermittent complete heart block.

\section{CASE REPORT}

A 24-year old male patient was brought into the emergency room (ER) via the Emergency Medical Services Team, complaining of abdominal pain. The pain was colicky in nature, localised in the right upper quadrant (RUQ) and reflected to the back. The onset of pain began three hours after eating doughnuts and cheese. He was nauseous and vomited non-bloody gastric contents four times. The patient's last bowel movement was two days prior, and was normal in colour and consistency. The patient was seen in the ER on various occasions prior to this due to biliary colic, thus a known diagnosis of cholelithiasis. A surgical consult was recommended and offered on prior visits, which he declined due to socioeconomic reasons. The patient was also aware of a pre-existing heart murmur since childhood, but had no medical records on hand. The patient had no appetite and no urinary complaints. $\mathrm{He}$ smoked twenty cigarettes per day, denied consumption of alcohol in the last two weeks and had no known drug allergies. Upon physical examination, the patient was afebrile, blood pressure was 105/70 $\mathrm{mmHg}$, pulse $70 / \mathrm{min}$, no heart murmur was detected, RUQ tenderness without guarding and a positive Murphy's sign. The remainder of the physical exam was without any further pathological findings. The electrocardiogram (ECG) was recorded in a sinus rhythm 70/min. Radiography of the abdomen was without abnormalities. Laboratory studies revealed hepatic enzymes to be within normal limits, and the parient's bilirubin levels, C-reactive protein and leukocytes were only mildly elevated. An ultrasonography of the abdomen revealed that the gallbladder was 8 $\mathrm{cm}$ in length, with the wall thickening and findings of pericholecystic fluid. Multiple concrements were present in the gallbladder. A follow-up ECG revealed a complete atrioventricular block with a ventricular frequency of $60 / \mathrm{min}$ which was not present earlier. This prompted an emergency cardiology consult. The cardiologist placed a temporary heart pacemaker via the femoral vein providing optimal ventricle stimulation. An emergency laparoscopic cholecystectomy was performed without any complications. The byopsy confirmed gangrenous acute cholecystitis. Following the surgical procedure the patient was in sinus rhythm and, the temporary pacemaker was thus removed. Postoperative recovery was without any pathological find- 
ings. An echocardiogram revealed a trivial mitral-valve regurgitation, an ejection fraction of $64 \%$ and all other parameters were within normal range. The Holter ECG monitor recorded a sinus rhythm of 69 beats per minute and detected an insignificant number of single monomorphic ventricular extrasystolic contractions, with no pauses in electroconductivity. The patient recovered quickly and completely from surgery without any residual sequelae.

\section{DISCUSSION}

Reflex bradycardia was described in two patients in 1971 with acute cholecystitis.

(4) Since then, more electrocardiographic changes and even angina pectoris have been linked to acute gallbladder disease, presumably because of a vagally-mediated cardiobiliary reflex. (5) A similar case to our male patient was recorded in 2009, in a patient who had three syncopal episodes while awaiting surgery. Telemetric ECG recordings revealed an episode of complete heart block without an escape rhythm for a duration of 9 seconds. The patient completely recovered after cholecystectomy was performed without recurrence of syncope or ECG abnormalities. (6) Since cardio-biliary reflex is triggered by pain in the gallbladder via an autonomic vagal innervations, and may occur in the presence or absence of stones in the gallbladder, it has thus been documented in acalcalous cholecystitis. (7) It is important to keep in mind that unexplained sinus arrest and bradycardia in a patient with abdominal pain should alert the possibility of cholecystitis. (7) In patients with known coronary artery disease, signs and symptoms of gallbladder and heart disease may overlap, masking the diagnosis. Nonetheless, if a patient has an acute exacerbation of biliary tract disease and associated transient electrocardiographic changes with no concurrent cardiac complaints or abnormalities, the surgeon should not be discouraged from performing cholecystectomy based on a "questionable" electrocardiogram. Any delay in treatment while awaiting cardiac screening results may result in both cardiac and septic complications. (5)

\section{CONCLUSION}

Considering the patient's age, normal cardiac biomarkers tests and the disappearance of the arrhythmia after the removal of the gallbladder, it is postulated that this patient had a case of cardio-biliary reflex. Special attention should be given to patients with acute cholecystitis experiencing concurrent ECG changes. Acute cholecystitis is a treatable cause of a newly diagnosed AV block and an immediate cholecystectomy should not be deferred.

\section{REFERENCES}

1. Friedman GD. Natural history of asymptomatic and symptomatic gallstones. Am J Surg. 1993;165:399-404.

2. Indar AA, Beckingham IJ: Acute cholecystitis. Br Med J 2002; 325: 639.

3. Hayden GE, Brady WJ, Pollack M, Harrigan RA. Electrocardiographic manifestations: Diagnosis of atrioventricular block in the emergency department. J Emerg Med. 2004;26:95-106.

4. O’Reilly MV, Krauthamer MJ. “Cope's sign” and reflex bradycardia in two patients with cholecystitis. Br Med J. $1971 ; 2: 14$.

5. Krasna MJ, Flancbaum L. Electrocardiographic changes in cardiac patients with acute gallbladder disease. Am Surg. 1986;52:541-3.

6. Franzen D, Jung S, Fatio R, Brunckhorst CB. Complete atrioventricular block in a patient with acute cholecystitis: a case of cardiobiliary reflex? Eur J Emerg Med 2009;16:346-7.

7. Lau YM, Hui WM, Lau CP. Asystole complicating acalculous cholecystitis, the "Cope's sign” revisited. Int J Cardiol. 2015;182:447-8. 